\title{
How and Why We Need to Capture Tacit Knowledge in Manufacturing: Case Studies of Visual Inspection
}

\author{
T. L. Johnson*, S. R. Fletcher, W. Baker, R. L. Charles \\ Industrial Psychology and Human Factors Group, \\ School of Aerospace, Transport and Manufacturing \\ Cranfield University, \\ Bedford, MK43 OAL
}

*Corresponding Author: t.l.johnson@cranfield.ac.uk

\begin{abstract}
Human visual inspection skills remain superior for ensuring product quality and conformance to standards in the manufacturing industry. However, at present these skills cannot be formally shared with other workers or used to develop and implement new solutions or assistive technologies because they involve a high level of tacit knowledge which only exists in skilled operators' internal cognitions. Industry needs reliable methods for the capture and analysis of this tacit knowledge so that it can be shared and not lost but also so that it can be best utilised in the transfer of manual work to automated systems and introduction of new technologies and processes. This paper describes two UK manufacturing case studies that applied systematic task analysis methods to capture and scrutinise the tacit knowledge and skills being applied in the visual inspection of aerospace components. Results reveal that the method was effective in eliciting tacit knowledge, and showed that tacit skills are particularly needed when visual inspection standards lack specification or the task requires greater subjective interpretation. The implications of these findings for future research and for developments in the manufacturing industry are discussed.
\end{abstract}

\section{Keywords}

Tacit knowledge, Visual inspection, Task analysis

\section{Introduction}

Visual inspection (VI) is a traditional manual activity that involves careful and critical assessment of an object with reference to a predefined standard (Drury and Watson, 2002; Drury and Dempsey, 2012; See, 2012). In manufacturing, VI is used to identify and diagnose defects, which is essential for ensuring products meet satisfactory quality standards (Garrett, et al., 2001). Despite typical error rates of between 20\% and 30\% (Drury and Fox, 1975), human VI has remained essential in manufacturing because the accuracy and efficiency of human visual acuity has remained superior to the visual inspection capabilities offered by available automated alternatives. Thus, although highly labour-intensive, VI continues to be particularly important in safety critical and high value manufacturing (HVM) processes where the consequences of missed defects are of a higher cost for both human and commercial reasons, e.g. "injury, fatality, loss of expensive equipment, scrapped items, rework, or failure to procure repeat business" (See, et al., 2017, p. 262). As visual inspection is highly skilled, best practice knowledge and techniques are valuable, and as it is labour-intensive it is a prime candidate for process improvement / automation to enhance process efficiency in the future. 
Visual inspection relies on 'tacit knowledge': an intrinsic understanding of how things work and are organised which enables humans to intuitively produce strategies and solutions in new circumstances (Reber, 1989). Whereas 'explicit knowledge' can easily be described, aggregated, codified and catalogued in written instructions for formal learning, tacit knowledge is less tangible. It is the personal and contextual awareness that we typically keep in our mind and its cognitive processes that is difficult to capture, classify or communicate, and typically "can only be acquired through practical experience" (Lam, 2000). Unlike explicit knowledge in training manuals and programmes, tacit knowledge is typically learned by observation, imitation and practice which is difficult to communicate (Smith, 2001). Therefore, tacit knowledge refers to the informal 'know-how' about how to do things that we all develop over time and experience, often unconsciously, which is typically retained in our individual memories but not formally recorded or shared.

In the context of manufacturing $\mathrm{VI}$ operations, there has been little research to specifically explore the role of tacit knowledge. We know that operators are typically provided with reference manuals and standard operating instructions (SOIs) which set out explicit knowledge about the task. However, it must be that operators build up their tacit knowledge 'know-how' about how to accurately identify / classify product defects through experience and repeated performance of $\mathrm{VI}$ tasks. Consequently, a considerable degree of more detailed contextual information about how best to detect and diagnose product defects must exist in skilled operators' personal memory stores and cognitive processes. Although it has been practical for this personal 'know-how' to remain undocumented and simply be applied as needed by skilled operators, there is a growing need for a formal method to capture and understand tacit knowledge in manufacturing $\mathrm{VI}$ tasks in order to transfer it to other human or automated processes. The following sections provide more detail to explain the three main reasons why tacit knowledge should be captured within manufacturing.

Firstly, tacit knowledge capture is needed for transfer to other operators. As it involves a far more comprehensive understanding of a task's content and protocol than could be derived simply from observation or design methods, sharing tacit knowledge with new or transitory members of the workforce is valuable. Without such an in-depth understanding of the "knowhow' that experienced operators employ to identify and diagnose defects, the formal training given to new operators is obviously more limited. Capturing this knowledge is going to be increasingly important as workforce mobility continues to rise (Favell et al., 2007; Pitts and Recascino Wise, 2010). Organisations will need to rely less on the well-established experience and skills of long-term workforces and, instead, rely more on utilising the capabilities of available less-experienced personnel. Thus, to effectively transfer the task procedure in instruction and training programmes an in-depth understanding of how the task is performed, in detailed steps, is inevitably going to be of great benefit. Without in-depth tacit knowledge the nuances of experienced 'know-how' will not be transferrable to transient and evolving workforces which is highly likely to impact negatively on production performance and efficiency.

Secondly, the capture of tacit knowledge is also going to be important to inform the design of new and emerging technologies. Advances in vision and sensing technologies are going to offer new opportunities for the automation and digitisation of VI processes (Caggiano, et al., 2015; Huang and Pan, 2015; Borrmann et al., 2016). However, in order to be effective these new systems will not be designed to merely replace original human activity because a) that would not utilise the new potential technological capabilities and b) it would not be possible to 
match human performance for many task steps, particularly those requiring flexibility and intelligence (Ding and Hon 2013; de Winter and Dodou, 2014). Therefore, the design of these new systems will not be based on a simple transfer of the original human task protocol but on a detailed analysis of it in which unnecessary or human-specific functions can be identified and eliminated or redesigned. This will require an accurate and thorough breakdown of the original manual work content which, therefore, should include tacit knowledge. Whether entirely new systems are being designed to replace human $\mathrm{VI}$, or whether systems are redesigned to augment / assist human operators in their manual VI activities, a thorough understanding of the original human performance and procedure will be needed to appropriately revise or reallocate functions.

Thirdly, tacit knowledge applied in VI manufacturing tasks needs to be captured to optimise the introduction and implementation of new systems and processes. It has long been recognised that the success of new industrial systems and technology can be highly dependent on how well their design and application has included consideration of human / social factors (Chung, 1996; Battini, et al., 2011). Clearly then, a good understanding of key human / social requirements and work activities is needed at the design stage so that key factors which will enhance a new system's introduction and operational effectiveness can be considered and included (and so that factors which are likely to have negative impacts on its success can be avoided). This means it is not only important that the manual work content of new systems is derived but that the wider human / social impacts are accurately predicted. Thus, to improve the likelihood of successful implementation of new systems, particularly with greater levels of automation and workforce diversity, there is a need for a reliable method to capture and analyse the tacit knowledge underlying VI work so that key barriers and enabling factors can be considered.

As the above examples illustrate, there is a need to capture tacit knowledge to accommodate forthcoming industrial challenges such as the evolving requirements of mobile workforces, advancing technology design, and effective new system implementation. To meet these challenges a reliable method for capturing, understanding and sharing tacit knowledge in manufacturing VI work is needed. This paper describes two case studies that have been conducted to address this industrial problem. First we present a selection of relevant background literature on the topic of VI in manufacturing.

\section{Background}

\subsection{The Process of Visual Inspection}

The manual process of VI involves five principal steps: Set up, Present, Search, Decide and Respond (Drury and Watson, 2002; Drury and Dempsey, 2012). Of these five steps, the 'search' and 'decide' activities appear to have received the most interest in previous investigations, probably because they are most cognitively complex and error-prone (Rao, et al., 2006; Drury and Dempsey, 2012; See et al., 2017). The 'search' step of VI has been found particularly time-consuming and prone to error, particularly in relation to omissions where a defect is missed rather than 'commissive errors' or false alarms (See, 2012). VI searching tends to not only involve visual skills but also the scrutiny of other sensory cues, such as touch and sound for tactile and auditory feedback (Garrett, et al., 2001). In manufacturing VI tasks, operators are often provided with additional equipment to assist their 'search' accuracy, such as additional lighting and magnifying devices (Charles et al., 2015). 
Although 'search' and 'decide' activities are totally independent processes (Spitz and Drury, 1978), if a search has led to identification of an anomaly or imperfection the inspector will then proceed to the 'decide' step to determine its type / class and the subsequent action that needs to be taken. In manufacturing VI tasks, the 'decide' step will require examining the object with reference to pre-defined standard criteria which is typically provided in reference manuals in written and graphical form. In addition, measurement equipment may be provided for the operator to more accurately classify the defect and its severity, and determine the action that needs to be taken. However, despite human sensory capabilities skill and the provision of equipment aids, there are still a number of factors which may impair VI.

\subsection{Factors that Affect Visual Inspection}

A great deal of literature from research studies has examined $\mathrm{VI}$ in manufacturing contexts, and more recently in relation to the development of new automated inspection systems (e.g. Golnabi and Asadpour, 2007; Lin, 2007; Lyu and Chen, 2009; Kumar and Kannan, 2010; Sun, et al., 2010; Mar, et al., 2011; Ravikumar, et al., 2011; Sun, et al., 2012; Huang and Pan, 2015; Mumtaz, et al., 2012; Caggiano, et al., 2015). There appears to have been little or no dedicated exploration of tacit knowledge in these various studies. However, in a wide review of the visual inspection research literature, See (2012) compiled a table listing the wide range of factors have been identified as influential to VI performance across different types of production (Table 1).

Table 1: Identified Factors that Impact on Visual Inspection Performance (from See, 2012)

\begin{tabular}{lllll}
\hline Task & Individual & Environmental & Organisational & Social \\
\hline Defect Rate & Gender & Lighting & Management Support & Pressure \\
\hline Defect Type & Age & Noise & Training & Consultation \\
\hline Defect Salience & Visual Acuity & Temperature & Retraining & Isolation \\
\hline Defect Location & Intelligence & Shift Duration & Instructions & Communications \\
\hline Complexity & Aptitude & Time of Day & Feedforward information & \\
\hline Standards & Personality & Vigilance & Feedback & \\
\hline Pacing & Time in Job & Workplace Design & Incentives & \\
\hline Multiple Inspections & Experience & & Job Rotation & \\
\hline Overlays & Visual Lobe & & & \\
\hline Automation & Scanning Strategy & & & \\
\hline & Biases & & & \\
\hline
\end{tabular}

These task, individual, environmental, organisational, and social factors will undoubtedly be relevant in varying degrees across specific manufacturing environments and VI processes. The enormous number of potential features that these factors comprise is too large for review here, and many are not directly relevant to tacit knowledge. However, some factors are especially and directly relevant to the development and application of tacit knowledge in manufacturing $\mathrm{VI}$ tasks and these will be discussed: Task - complexity, standards and pacing; Individual - scanning strategy; Organisational - training.

\subsubsection{Task factors}

As shown in Table 1, task-related factors are determined by the features of the particular object that is being inspected and its defects. There is an obvious relationship between the complexity of an object's visible features and the complexity of the task of inspecting it. Task complexity is a highly important factor across different conditions and environments, and is a 
function of these features. Complexity is defined by the physical nature of the object being inspected (e.g. size, shape, surface texture, condition) as well as the defects that are present or potentially present on the object (e.g. number of defects, number of different defect categories) and their distribution (Gallwey and Drury, 1986; Rao, et al., 2006).

Greater intricacy of an object's features has been found to negatively affect VI (Harris, 1969), and the condition of the object's surface can mask defects and complicate visibility, not only if unclean but if uneven or textured (Drury and Clement, 1978). The number and variability of defects on an object also affect VI task complexity and performance. For example, a higher number of defects has been found to increase levels of cognitive demand (Wickens and Carswell, 2012). Laboratory studies have also found that as the number of fault types under scrutiny increases the degree of fault detection decreases, and that inspection for multiple faults is found more difficult than inspecting for single faults especially if they appear physically similar (Gallwey and Drury, 1986; Rao, et al., 2006). These impacts on task demand and complexity are important given that visual inspectors often need to look for several types of defects, of varying size and shape, at the same time as part of their work. As it is not possible to eliminate these factors it is likely that inspectors, over time, develop ways to compensate for component and defect intricacy. No details about how inspectors may do this were found within the literature, however it is likely that people develop compensatory techniques or 'scanning strategies' in order to execute their tasks successfully - and this becomes tacit knowledge over time as inspectors put them into practice in their day to day work.

Inspection standards are another factor common to VI across conditions. Well written standards can reduce cognitive demand and the need to rely on subjective judgements, particularly when they include visual aids (pictures or photographs), and / or computer based prompts (Drury and Watson, 2002). However, the provision of written / graphical information can also contribute to task complexity (Gallwey and Drury, 1986) and, unsurprisingly, their complexity has an impact on both 'search' and 'decide' performance (Rao et al., 2006). Most inspection standards and defect classification systems are based on several factors such as defect type, defect location, and defect size but those that are poorly written and ambiguous incur greater cognitive strain for judgement and decision making (Gramopadhye, et al., 1997, Rao, et al., 2006). If inspection standards do not clearly distinguish different defect characteristics and types this leads to increased task complexity, fatigue and workload, along with lower accuracy of defect identification (Wickens and Carswell, 2012).

Unclear definitions and instructions for $\mathrm{VI}$ also tend to encourage inspectors to develop their own subjective assessment criteria, which is not only at risk of being incorrect but can also drift over time and lead to greater inconsistency in performance (Juran and Gryna, 1988). Decisions based on subjective perception and previous experience of defects have been found to have lower reliability (Garrett, et al., 2001; Laofor and Peansupap, 2012) which may be due to increased demands on the memory in a process that is already reliant on an extensive use of short and long term memory (Gallway, 1998). However, it must also be considered that these personal methods and criteria could be equally efficient and correct, given that people remain superior to any automation for performing $\mathrm{VI}$ and decision-making activities (Thapa, et al., 1996), adapting to unforeseen events (Drury and Watson, 2002), and utilising highly responsive tactile senses (Garrett, et al. 2001; Heidl, et al., 2013). The development of these abilities and knowledge of both the part and defects over time is the formation of tacit knowledge. As mentioned above, the problem that the development of tacit knowledge poses is that if this knowledge and understanding is not passed on to other 
inspectors, or captured and recorded, it may be lost. Additionally, if captured, this information could be used to inform the design of new and emerging technologies which would aid inspectors in their inspection process.

The time and pace of $\mathrm{VI}$ tasks is another factor relevant to all $\mathrm{VI}$ tasks. The time allowed for VI tasks has a significant impact on performance (Garrett, et al., 2001) and this corresponds with the negative impact of work pace on industrial task performance in general (e.g. Dudley, 1963; Lin et al., 2001). However, no evidence was found in the literature regarding the potential for tacit knowledge and experience to counterbalance the negative effects of limited time and task pace.

\subsubsection{Individual factors}

Almost all of the range of potential individual factors listed in Table 1 are defined by people's specific characteristics and experiences and, therefore, are context-specific. However, 'scanning strategies' that people use in VI tasks are most relevant to the role of tacit knowledge in manufacturing across individuals. Courtney and Guan (1998) found that VI search strategies vary greatly across individuals and tasks, manifest in whether the search is random, systematic, or random with some controlled overlap (where the inspector avoids scanning already searched areas, but may overlap due to memory loss). Random searches that lack pattern have been found to not only take longer than a more systematic search but also be more vulnerable to defects being missed by the inspector (Gramopadhye, et al., 1997; Tetteh and Jiang, 2006). Systematic searching, on the other hand, has been found to result in better performance as measured by a significantly greater detection of defects, a quicker search (Gramopadhye, et al., 1997; Clemons, 2013), and increased perceptual sensitivity for defects (Watts, 2011).

Although Drury and Watson (2002) state that individuals naturally tend to follow an unstructured pattern when searching an object, Drury and Chi's (1995) laboratory experiment findings indicated that the design of an object (in their example a circuit board) may encourage and assist participants to adopt a systematic VI approach. If the inspection task / object does not characteristically lend itself to a systematic approach then it is possible for the inspector to use cognitive feedback to apply a systematic search, by applying information about scan path and coverage that they may have acquired in training (Gramopadhye, et al., 1997) or through experience in normal working practices. Clearly then, search or scanning strategies are a significant part of $\mathrm{VI}$ tacit knowledge development and are likely to be particularly efficient and accurate if developed directly from on the job experience of overcoming task factors that present difficulties mentioned in Section 2.2.1. In order to ensure this valuable knowledge can be shared amongst manufacturing work inspectors and used for successful process / automation design there is a need to codify the current visual inspection techniques that inspectors employ.

\subsubsection{Organisational factors}

In the list of organisational factors that are presented in Table 1, training is directly relevant to the development of tacit knowledge in manufacturing VI tasks. Operators in manufacturing environments invariably receive some form of training which will consist of aggregated and codified explicit knowledge but, as described in the introduction to this paper, tacit knowledge will be developed over time via more informal practical experience. Unsurprisingly, training 
(including retraining) can significantly improve inspection performance (Drury and Watson, 2002) and a consistent training programme can enhance the speed, accuracy, and performance of visual inspectors including those new to the role (Gramopadhye, et al., 1997). Experimental research evidence also suggests that training which requires an active response may be more effective than a passive programme (Czaja and Drury, 1981) and in one industrial case study a significant drop in error rates, scrap, and rework was recorded after a modular active response training programme was introduced (Kliener and Drury, 1993). However, whilst it is clear that well-designed training may optimise VI performance there does not appear to have been any exploration to determine the potential enhancement that might be made if tacit knowledge of experienced workers could be captured and disseminated in formal training.

\subsection{Tacit knowledge}

As described, tacit knowledge is the 'know-how' about an object or subject that people develop through personal experience and not formal training, but rarely articulate because it exists in memory and cognitive processes and "often resembles intuition" (Smith, 2001, p.314).

In the workplace, the intangibility of tacit knowledge and the difficulty of converting it into explicit knowledge means organisations may lose critical information and best practice when skilled employees leave (Smith, 2001). It would, therefore, be advantageous to systematically capture and analyse experienced workers' tacit knowledge, so that it could be routinely disseminated via work standards and formal training. In the context of manufacturing $\mathrm{VI}$ work, capturing tacit knowledge would be particularly desirable to address the contemporary industrial challenges set out in the introduction to this paper, so that more effective methods of defect identification and classification protocols may be transferred to other personnel and to the design of new systems and implementation strategies.

Attempts have been made to develop effective methods for the capture and analysis of tacit knowledge and skill. For example, Phipps, et al., (2011) applied the skill, rule and knowledge (SRK) framework developed by Rasmussen (1983; 1986) to traditional hierarchical task analysis (Kirwan and Ainsworth, 1992) in order to identify tacit cognitive task elements. Everitt, et al., (2015) also applied this method to capture and classify the knowledge and skills applied in complex manufacturing task performance for the purposes of automation design. In both of these examples the SRK framework is used for decomposition of the task so that every individual step of activity in human task performance can be classified according to one or more of three knowledge levels which progressively require more cognitive effort:

Skill: behaviour involves an immediate recognition of a situation, followed by a preprogrammed physical response (Embrey, 2005). Performance is smooth and automatic, requiring little attention because the required actions are understood in advance (Rasmussen, $1983 ; 1986)$.

Rule: behaviour is guided by more conscious control in the form of stored rules derived first hand from prior experiences or conscious problem solving (Rasmussen, 1983; 1986). The individual is aware that there may be alternative courses of action, and that a choice may need to be made between them (Elliott, 2005). 
Knowledge: behaviour corresponds to a predominantly cognitive stage of a task, often in unexpected situations, which requires a higher degree of conscious control than for rule or skills based behaviours, and analysis based on an understanding of explicit goals.

The SRK framework enables systematic analysis of task performance and, as shown by the definitions above, it specifically allows interpretation of the degree to which components of a task involve cognitive effort via automatic or conscious activities. Additionally its presentation of skill, rule, and knowledge as types of performance, rather than linear progressions of skill acquisition as seen with 'Phases of skill acquisition' (Fitts, 1964) or the 'Acquisition of cognitive skills' (Anderson, 1982) models, enables classification of task steps within a process. Allowing the identification and deeper investigation of specific aspects of a task that are more prone to the development of skill and therefore tacit knowledge. Consequently, the SRK framework appears to be an ideal framework to apply in the capture of tacit knowledge, as a result this study will follow the previous work by Phipps, et al., (2011) for task decomposition.

Having outlined the importance of capturing tacit knowledge in VI task performance, this paper now describes the method used in two case studies that explored tacit knowledge using the SRK framework. Specifically, this research set out to determine whether application of the SRK framework to traditional hierarchical task analysis would be an effective means of eliciting tacit knowledge used in visual inspection.

\section{Method}

Two UK manufacturing facilities were nominated for the two field studies by the high-value aerospace manufacturing company who sponsored this work because they both comprised VI workforce units that were critical to production. One facility (Area A) was responsible for new component production, so VI is conducted there to check for any flaws that may have occurred during the production process and to ensure production quality. The other facility (Area B) houses in-service product maintenance activities where in-use components are returned and disassembled for VI to be conducted to check for any degradation or damage, so the part can be either repaired or replaced. By investigating tacit knowledge in visual inspection used in these two different facilities it was possible to identify commonalities and differences in the development of tacit knowledge.

\subsection{Participants}

Area A: Twelve participants were interviewed across two shifts (six participants in each shift), ten of which were female and two were male. Data were collected over 7 hours each day, with $31 / 2$ hours spent with each shift. One inspector interviewed was a trainee, another was a newly qualified inspector, and the rest had at least one year of experience.

Area B: Nine participants were interviewed across two shifts, all were male and all had at least a year of experience. Data were collected over 7 hours each day, with $3 \frac{1}{2}$ hours spent with each shift.

The age of participants interviewed was not collected in this study, due to known sensitivities within this organisation. However, each inspector's years of experience in the job role were collected as this is relevant to the development of tacit knowledge. All available inspectors at the time of the study took part.

\subsection{Research Ethics}


The research was approved by the Cranfield University Research Ethics Committee, and performed in accordance with the Cranfield Research Integrity Policy and the British Psychological Society's Code of Human Research Ethics (2014).

Inspectors were notified in advance by the organisation's management. Researchers then gave a formal briefing to each participant in the study to provide information about the nature of the study and its requirements, and data processing protocols, before participants' informed consent was obtained.

\subsection{Procedure}

Prior to formal data collection the researchers were given tours of both areas. This provided the researchers with a basic understanding of the manufacturing and VI processes to inform questioning during data collection. Data were collected during twelve days of site visits: four days spent in Area A comprising of two sessions of two days each, and eight days in Area B comprising of one two-day, and two three-day visits, ensuring sufficient coverage of the different shifts.

In this study an ethnographic approach was adopted, with data collected through observations and unstructured interviews. No formal interview schedule was developed prior to data collection. The interviewers asked the inspectors to talk through their inspection tasks as they performed them to demonstrate their VI procedures and explored additional questions as they emerged. During the first session in both areas, the inspectors explained and demonstrated their VI tasks and processes, responded to unstructured questioning, and provided background information on the content of the Standard Operating Instructions (SOIs) and reference manuals. Between the data collection sessions, field notes were reviewed by the researchers, individually and together, to stimulate questions for subsequent data collection events, to optimise available time during data collection, and to refine the selection of the analysis categories. The review between operators ensured consistency between the interviewers and the questions they asked. Observation and interview sessions were run until data collection redundancy was reached. Task documentation including SOIs and manuals were additionally reviewed after data collection to identify where tacit knowledge may have developed to compensate for limitations in the documentation.

The VI tasks being examined in each case study involved teams of inspectors working on the identification, classification, and 'sentencing' (determining the remedial action needed) of defects in aerospace components. When examining these components the inspectors search for obvious defects and known problem areas that may lead to the object being scrapped, such as large structural defects. A more in-depth visual search then follows with any defects identified and marked up. Some inspectors would 'sentence' defects to determine what remedial action was needed immediately after detection, whereas others would conduct their sentencing evaluation at the end of the search process. This variation would depend on how the inspectors had been trained and the particular facility in which they were working. For example, due to the smaller size of the components inspected in Area $A$ the sentencing procedure was more likely to be completed at the end of the inspection process whereas within Area $B$ there were instances of sentencing being conducted as defects were identified due to the much larger size of the components. Sentencing decisions were made with reference to formal inspection standards which are provided to inspectors to classify types of defects and features, such as acceptable or unacceptable size and / or shape, and often include images 
or diagrams. Three sentencing outcomes were used within both facilities: acceptable, rework, and scrap. If an object was sentenced as a rework it would be inspected again when it was returned to ensure the defect had been removed and that the component was still acceptable with regards to its overall shape.

The final visits in each area were used to validate the data that had been gathered, and for this inspectors reviewed the analysis output that had been developed by the researchers following data collection. This process enabled inspectors to substantiate whether the data had been correctly recorded or point out any errors or omissions that needed amendment, thereby enabling the researchers to be confident that the data analysis had produced an accurate representation of the VI processes.

\subsection{Analysis}

The data from the interviews and observations were analysed using Hierarchical Task Analysis (HTA), and Task Decomposition (TD) to distinguish between procedural and tacit knowledge and to identify skill, rule, or knowledge based performance. It is not possible to include whole examples of either the HTA or the TD in this paper due to their size and the commercial sensitivity of the data included in them. However, a sanitised section of the TD from each area can be found under the Task Decomposition subheading (Tables 2 and 3). These techniques are also now described.

\subsubsection{Hierarchical Task Analysis}

In this study the HTA method was used for VI task analysis because it enables the logical deconstruction of tasks into physical and cognitive components (Kirwan and Ainsworth, 1992) following a systematic procedure. The technique is used to break a task down according to its series of goals and sub-goals. In this study this method was used for VI task analysis as follows:

- Task: Visual Inspection.

- Overall goal: To identify and sentence defects on individual parts.

- Sub-goals: The goal was broken down into further sub-goals, which collectively form the task components that need to be performed in order to achieve the overall goal.

- Operations: The sub-goals were broken down into a hierarchy of operations in turn to identify the actions which need to be performed to achieve the overall goal. The nesting of operations is used as a method to group operations together that are part of the same task, they help to categorise parts of the task, therefore they are not perceived as fractions or parts of a higher operation, but operations in their own right that are required in order to successfully complete a task.

To keep track of the hierarchical levels a rational numbering system was used with the overall goal numbered 0 , sub-goals numbered $1-\mathrm{n}$, and operations then numbered using their subgoals to list their order of occurrence, and then their own number which depends on their order in the sequence (e.g. 1.1 - 1.3.1). The data collection for the HTA continued until operations had been re-described by the operator(s) to the level of description where the steps of a task were completely clear and understood by the researchers; so that an operator could carry out the task and meet the goal, in accordance with Piso's (1981) methodology. In order to provide a better representation of the cognitive task components, TD was then used to extend the HTA. 


\subsubsection{Task Decomposition}

Task decomposition was then applied to the HTA. This technique is a structured expansion of the HTA into a series of more detailed statements identifying specific aspects of the task. The series of detailed statements are grouped under subheadings designed to decompose the task elements. To select the TD categories, reference was made to the categories suggested by Kirwan and Ainsworth (1992). Additionally, key factors and issues that had been identified in the literature on $\mathrm{VI}$ and the nature of human skill was considered. The selection of categories (and HTA) was refined as observations and data collection progressed. The TD categories and corresponding descriptions are as follows:

- Purpose: The reason for a particular operation. This category was included to improve the readability and comprehension of the HTA and decomposition.

- Cues: The cues and feedback that the inspectors use to identify and sentence defects. How does the inspector attend and respond to these cues (whether consciously or unconsciously) during the task?

- Decision: The decisions made by the inspector. Given a particular cue, or pattern of cues, what decisions does the operator make in order to perform the task successfully?

- Performance level: The category of operator performance according to the SRK framework (Rasmussen, 1983). Through identification of task components, does performance require a level of conscious control that signifies rule or knowledge-based behaviour (explicit or declarative knowledge) or is it implicit and skill based (tacit knowledge)?

- Likely errors and error correction: The types of error and remedial actions that inspectors make. What are the likely errors that could be made by the inspectors and how are these corrected?

- Critical values: The values that may potentially provide a basis for rule-based performance. Are there any particular critical values relating to the inspection and diagnosis of an object / component which may influence performance or errors?

- Variations between individuals and protocols: The differences between task procedures used by inspectors and deviations from formal standards. How do inspectors' VI strategies and performance vary and how different are they from the recommended procedures in SOls?

These categories enabled a systematic analysis of each task step, thereby providing an indepth summary that included skill, rule or knowledge categorisation. Tables 2 and 3 show a sanitised section of the TD for Area A and B. Each instance of skill, rule, and knowledge could then be tallied, divided by the total number of performance levels (41 for Area A, and 89 for Area B) and multiplied by 100 to identify the percentage of each type of categorisation and the breakdown by $\mathrm{VI}$ step, which will now be reflected in the results section.

Table 2: Area A Task Decomposition Subsections (Limited to Two Tasks)

\begin{tabular}{llc}
\hline $\begin{array}{l}\text { HTA Goals, } \\
\text { Sub-goals, } \\
\quad \text { and }\end{array}$ & \multicolumn{1}{c}{ HTA Task Component } & $\begin{array}{c}\text { Performance } \\
\text { Level }\end{array}$ \\
Operations & & \\
0 & Identify and classify defects on components. & Rule \\
5.2 & Inspect each component individually & Skill \\
5.2 .1 & Move component so that all surfaces are inspected under different angles of light & Skill \\
5.2 .2 & Check each surface in order & Rule \\
5.3 & Mark any defects & Rule \\
\hline
\end{tabular}




\begin{tabular}{|c|c|c|c|}
\hline 6 & Assess defects & & \\
\hline 6.1 & Assess each defect in accordance with relevant documentation & & \\
\hline 6.1 .1 & Determine whether the defect will be machined off the finished component & Skill & Rule \\
\hline 6.1 .2 & Determine the type of defect & Skill & Rule \\
\hline 6.1 .3 & Determine acceptability of defect & Skill & Rule \\
\hline
\end{tabular}

Table 3: Table 1: Area B Task Decomposition Subsections (Limited to Two Tasks)

\begin{tabular}{llrl}
\hline $\begin{array}{l}\text { HTA Goals, } \\
\begin{array}{c}\text { Sub-goals, } \\
\text { and }\end{array} \\
\text { Operations }\end{array}$ & HTA Task Component & $\begin{array}{c}\text { Performance } \\
\text { Level }\end{array}$ \\
\hline 0 & Inspect and sentence components & \\
\hline 7.3 & Inspect to the level dictated by documentation & Rule & Skill \\
\hline 7.4 & Write down a list of the defects found noting where they are & Rule & Skill \\
\hline 7.4 .1 & Match the defect on the component to the defects outlined in the manual & Rule & Skill \\
\hline 7.4 .2 & Sentence these defects based on the sentencing criteria within the documentation & Knowledge & S \\
\hline
\end{tabular}

\section{Results}

Figures 2 and 3 show the presence of skilled performance and, therefore, tacit knowledge within visual inspection. This provides evidence of the effectiveness of HTA and TD in identifying tacit knowledge. Within both Figures the SRK breakdown is presented for each of the VI processes outlined in the functional model (Drury and Dempsey, 2012; Rao et al. 2006). This enables the identification of tacit knowledge within the specific VI task's processes: 'set up', 'search', 'decide' and 'respond' processes. The 'present' step of the model was not included as this was not identified as part of the inspection process used by inspectors in either of the Areas.

\subsection{Area $A$}

Figure 2 presents the overall breakdown of skill, rule, and knowledge used by inspectors throughout the VI process completed in Area A. Overall, $85 \%$ of tasks were identified as using procedural knowledge (knowledge and rule), with 15\% of tasks using tacit knowledge (skill). The higher percentage of procedural knowledge is unsurprising because the SRK framework is applied to observable behaviour and participant's verbal reports of their activities, and procedural knowledge is easier to verbalise than tacit knowledge. Out of the four steps of $\mathrm{VI}$, tacit knowledge was identified in only the 'search' and 'decide' steps of visual inspection. Within the 'search' step 30\% of the task used skill based behaviours, $50 \%$ rule, and $20 \%$ used knowledge. Knowledge based behaviour was not observed in any other VI steps within Area $A$, this suggests that there is a part of the 'search' task that requires a high degree of conscious control and analysis. Within the 'decide' steps less tacit knowledge was used compared to procedural knowledge, with a difference of $25 \%$. The 'respond' step for Area A shows $100 \%$ use of rule based information, indicating that the way the inspectors respond once they have identified and sentenced a defect is highly procedural. The 'set up' process is additionally highly procedural with $100 \%$ rule based behaviour observed. 


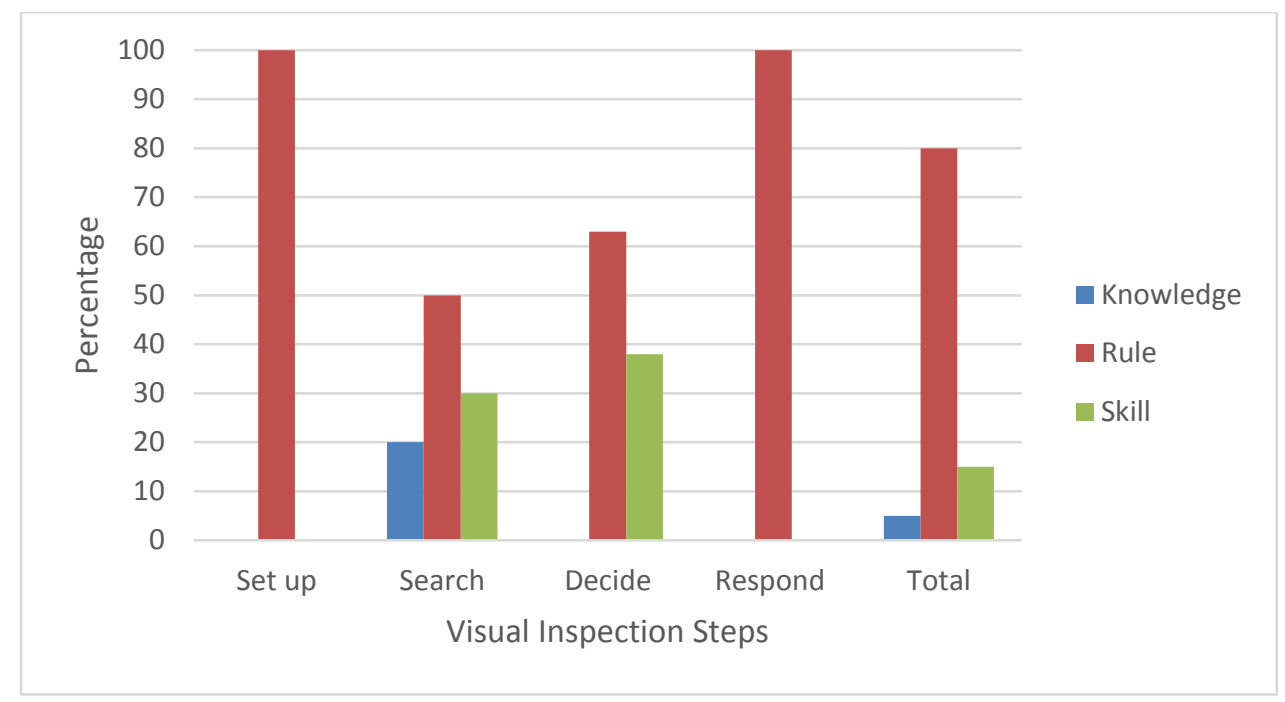

Figure 2: Area A Skill Rule Knowledge Breakdown by VI Steps (\%)

\subsection{Area B}

Within Area B the proportion of rule based tasks was $80 \%$ and $18 \%$ were skill based (Figure 3 ). Figure 3 shows that skill based behaviour, indicating the use of tacit knowledge, was required in three out of the four VI steps, and within 'set up' and 'decide' the amount of rule based behaviour was greater than tacit knowledge. However, the 'search' step analysis showed a $50 \%$ split between tacit and procedural knowledge. The significant implication of this finding is that if the tacit knowledge were to be lost the detail of half of the steps completed by inspectors during the 'search' phase of inspection would be lost with them. It is likely that the consequence of this would be a dramatic decrease in the effectiveness of defect identification.

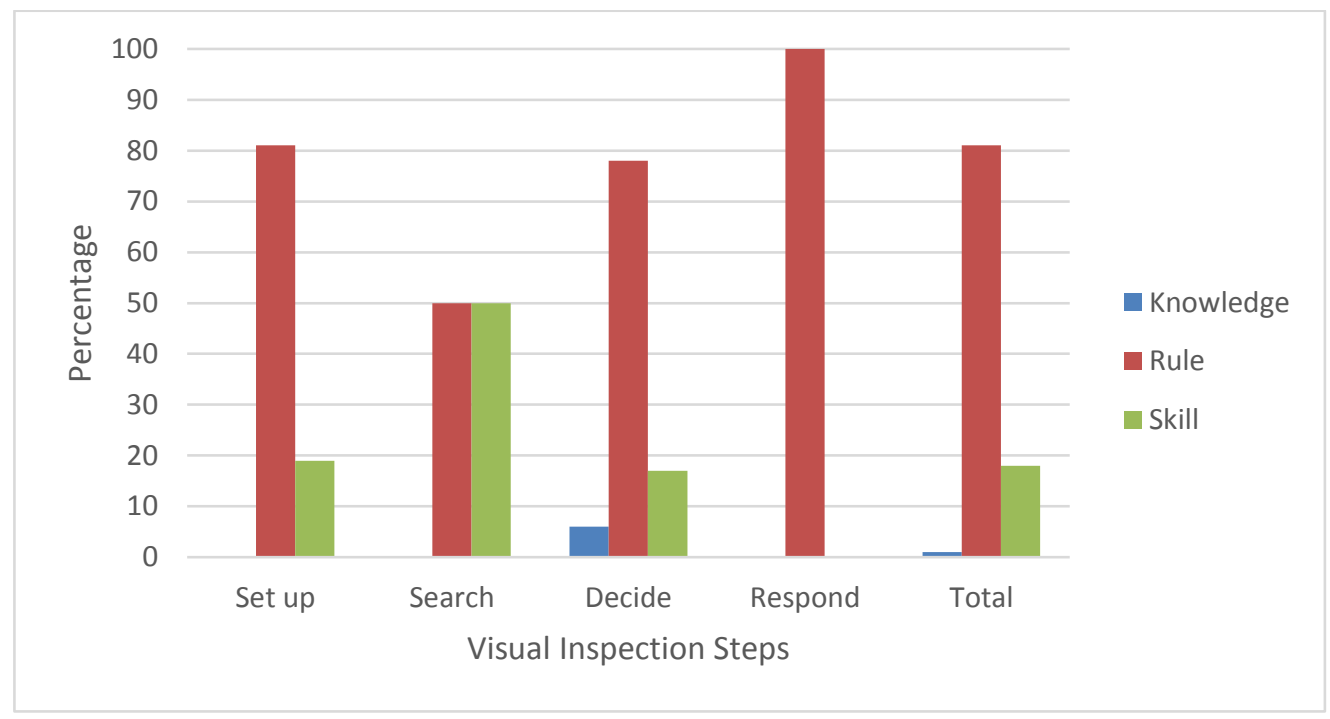

Figure 3: Area B Skill Rule Knowledge Breakdown by VI Steps (\%)

To summarise, tacit knowledge was found in the VI conducted in both facilities. Within Area A tacit knowledge was identified during the 'search' and 'decide' steps; and in the 'set-up', 'search', and 'decide' steps of VI for Area B. Illustrating that the occurrence of tacit knowledge 
in VI can vary from one facility to another even when part of the same organisation, and that tacit knowledge requires capture from all aspects of a company, not just a single facility.

\section{Discussion}

This paper describes two case studies that were conducted to address the industrial need for a method of tacit knowledge capture and analysis. Application of the combined HTA and TD techniques were successful in identifying the occurrence of tacit knowledge in manufacturing VI tasks being performed in operational UK facilities. The method showed the incidence of tacit knowledge in four of the five steps outlined in the functional model of VI (Drury and Dempsey, 2012; Rao et al. 2006) and that tacit knowledge is used widely in VI tasks. Moreover, results provide an insight into the important role of tacit knowledge and skill in mitigating factors that have been associated with VI performance impairments.

In both Areas inspectors were found to follow a systematic search pattern similar to that described by Gramopadhye, et al. (1997) which begins in the same location and follows the same path for each component. However, instead of using a prescribed pattern of inspection derived from formal training and instructions, the inspectors demonstrated that they had adopted patterns and strategies based on knowledge gained from their experience in normal working practices. In these developed methods, critical and known problem zones were inspected first which included areas that previously presented defects that were sentenced as scrap. In this way the inspectors' independent search strategies enhance inspection efficiency as they enable more immediate identification of scrappable defects before the entire component is subjected to a full and time-consuming inspection. However, these known problem areas did not appear to be recorded or formally presented to inspectors in the organisation's work standards which means this information would have to be stored in inspectors' memories and tacit knowledge. As mentioned in the literature, retaining this knowledge and applying it to the visual inspection process is likely to lead to increased task complexity for operators particularly when this process is still being applied as knowledge or rule based performances (Gramopadhye, et al., 1997). Indeed, it was observed that after known areas had been inspected both Area A and B inspectors followed a systematic inspection process which would reduce workload and ensure all steps are completed. This example shows that if this sort of information can be captured and provided in formal training it may reduce operators' cognitive load for this part of the inspection process.

In Area A the study showed that operators apply tacit knowledge and skills to adapt methods to combat task complexity caused by surface texture, as exposed by Drury and Clement's (1978) research, by adjusting components and moving them under light sources to increase visibility of defects. Task complexity is also likely to be heightened in Area A due to an extensive defect list which would be expected to contribute to increased cognitive demand (Wickens and Carswell, 2012) and, therefore, to missed defects (Gallwey and Drury, 1986; Rao, et al., 2006; Drury and Dempsey, 2012). Additionally, some pictures and descriptions in the inspection standards were found unclear and, in some cases, did not clearly distinguish between defect types which would compound task complexity and cognitive demand. However, the study found that this complexity may be counterbalanced by a collaborative and mentoring environment in Area $A$, as experienced inspectors were found to share their knowledge with less experienced inspectors. It is highly likely that this collaborative approach will reduce cognitive demand, improve standardisation, and help prevent the development of inaccurate subjective methods. It supports the way tacit knowledge is passed on within 
organisations (Smith, 2011) and constitutes one of See's 'social factors as shown in Table 1, demonstrating that even factors that do not appear to be directly related to tacit knowledge may contribute to its development and sharing amongst personnel, and may reduce the loss of unrecorded experiential knowledge.

As the components being inspected in Area B are in-service components they arrive dirty and, therefore, defects may be masked. However, here tacit knowledge was also found being applied to combat task complexity as the hand cleaning task prior to full inspection is used to complete a preliminary top-level inspection which pairs tactile and visual feedback to identify potential problem areas. This is highly likely to reduce the additional cognitive load caused by visual impairment from the dirt on the components' surface.

These results show that, over time, personnel observed in both case studies have developed solutions to limitations and challenges in their VI tasks which have developed into a range of tacit knowledge and skills. The elicitation and identification of tacit knowledge in these case studies verifies the findings from Phipps, et al. (2011) and Everitt, et al. (2015), and provides further evidence supporting the effectiveness of the task decomposition technique using Rasmussen's (1983 and 1986) framework. This method could therefore be used more widely within organisations to identify where task protocols and reference documentation can be improved. In particular, it demonstrates that it is possible to capture and codify tacit knowledge that has been built up from experience so that more detailed skills can be taught through formal learning as part of explicit knowledge. In future research it would be advantageous to explore more, or all, of the factors in See's compilation (Table 1) and to identify relationships between tacit knowledge / skills development and the performance of VI tasks, particularly with respect to the mitigation of performance constraints and complexities. For example, a limitation of this study is that age and experience levels of the participant sample were not considered relevant and age was not recorded, but future investigations could include analysis of these and other individual level factors in a wider sample to ascertain the impact of workforce characteristics. This sort of information could inform methods of induction and training but also the potential moderating or mediating effects of developmental factors such as experience and training programmes. Additionally, research exploring training and learning could be used to identify how and whether behaviours transition from knowledge through rule to skill, and whether there are types of activities more prone to transition than others, which would also be highly useful for the development of induction and recurrent training schemes.

The studies demonstrated the effectiveness of building analysis from qualitative ethnographic methods which provides rich and detailed subjective insight. However, this is a long and laborious research process which would not be efficient for industrial application. Thus, it would be advantageous if future work was undertaken to develop more objective practical methods for measuring tacit knowledge which could be used to complement and enhance subjective qualitative data.

For the manufacturing industry, the benefits of capturing the valuable tacit knowledge that currently only exists inside inspectors' cognitive processes is immense. This work has focused on investigations of visual inspection but the principles of tacit knowledge and skills capture are relevant to any tasks which involve a considerable degree of cognitive reasoning and experiential 'know-how'. To date, the highly developed skills of experienced operators are considered 'craft' based and intangible abilities but this work demonstrates the feasibility of applying a systematic method to reach hidden expertise. Application of this type of method 
could be critical to the development of effective future manufacturing system processes by enabling organisations to address the challenges of changing populations and technologies, and to allocate human skills appropriately.

\section{References}

Anderson, J.R., 1982. Acquisition of cognitive skill. Psychological Review. 89, 4, 369-406.

Battini, D., Faccio, M., Persona, A., Sgarbossa, F., 2011. New methodological framework to improve productivity and ergonomics in assembly system design. International Journal of Industrial Ergonomics, 41(1), 30-42.

Borrmann, D., Leutert, F., Schilling, K., Nüchter, A., 2016. Spatial projection of thermal data for visual inspection. Proceedings of the $14^{\text {th }}$ International Conference on Control, Automation, Robotics and Vision (ICARCV)

British Psychological Society, 2014. Code of Human Research Ethics. Retrieved $27^{\text {th }}$ June 2018, from https://www.bps.org.uk/news-and-policy/bps-code-human-research-ethics-2ndedition-2014

Caggiano, A., Caiazzo, F., Teti, R., 2015. Digital factory approach for flexible and efficient manufacturing systems in the aerospace industry. Procedia CIRP, 37, 122-127.

Charles, R.L., Johnson, T.L., Fletcher, S.R., 2015. The use of Job aids for visual inspection in manufacturing and maintenance. Procedia CIRP, 38, 9-93.

Chung, C.A., 1996. Human issues influencing the successful implementation of advanced manufacturing technology. Journal of Engineering and Technology Management, 13(3):283299

Clemons, A.J., 2013. Training methods for visual inspection tasks. Graduate Theses and Dissertations. 13005. Retrieved $27^{\text {th }}$ June 2018 , from http://lib.dr.iastate.edu/etd/13005

Courtney, A.J., Guan, L., 1998. Assessing search performance with a simulation model. Human Factors and Ergonomics in Manufacturing \& Service Industries. 8, 251-263.

Czaja, S.J., Drury, C.G., 1981. Training Programs for Inspection. Human Factors. 23, 4, 473484

Defence Science and Technology Organisation. 2005. Expert Decision-Making in Naturalistic Environments: A Summary (DSTO-GD-0429). Australia: Elliott, T.

de Winter, J.C.F., Dodou, D., 2014. Why the Fitts list has persisted throughout the history of function allocation. Cognition, Technology \& Work. 16, 1, 1-11

Ding, Z., Hon, B., 2013. Constraints analysis and evaluation of manual assembly. CIRP Annals - Manufacturing Technology. 62, 1, 1-4.

Drury, C. G., Fox, J. G., 1975. The imperfect inspector. In C. G. Drury \& J. G. Fox (Eds.) Human Reliability in Quality Control (pp. 11-16). London: Taylor \& Francis.

Drury, C.G., Chi, C.F., 1995. A test of economic models of stopping policy in visual search. IIE Transactions. 27 (3), 382-393. 
Drury, C.G., Watson, J., 2002. Good practices in visual inspection. Human Factors in Aviation Maintenance-Phase Nine, Progress Report, FAA Human Factors in Aviation Maintenance. Retrieved 27 $27^{\text {th }} \quad$ June $2018, \quad$ from https://www.researchgate.net/publication/267548062 GOOD PRACTICES IN VISUAL IN SPECTION? sg=nqBX5davu6eDbmmQRV0QqZ1vMZuAJeSBUi8 Ci0wdOCN1hrCa lyidOJ mIV9F860 4js5U20QW4MG5Pt9JlsH E LQ-USR6ojqdl4AM-.Vag8F0GXxlgG YN3kLi enG8q6uZ9i1qhY7r7CTr-m2RDZIIxEmbjT84RsFCRbvuBtNttTaSXEzZb992J2MSw

Drury, C.G., Clement, M.R., 1978. The effect of area, density, and number of background characters on visual search. Human Factors. 20, 597-602.

Drury, C.G., Dempsey, P.G., 2012. Human Factors and Ergonomics Audits, in G. Salvendy (Eds.) Handbook of Human Factors and Ergonomics, Fourth Edition. John Wiley \& Sons, Inc., New Jersey.

Dudley, N. A., 1961. The effect of pacing on worker performance. The International Journal of Production Research, 1(2), 60-72.

Embrey, D., 2005. Understanding human behaviour and error. Human Reliability Associates. $1,1-10$.

Everitt, J., Fletcher, S., Caird-Daley, A., 2015. Task analysis of discrete and continuous skills: a dual methodology approach to human skills capture for automation. Theoretical Issues in Ergonomics Science. 16, 5, 513-532.

Favell, A., Feldblum, M., Smith, M. P., 2007. The human face of global mobility: A research agenda. Society, 44(2), 15-25.

Fitts, P.M. 1964. Perceptual-motor skill learning. Categories of Human Learning. 8, 381-391.

Gallwey, T.J., 1998. Evaluation and control of industrial inspection: part II: The scientific basis for the guide. International journal of Industrial Ergonomics. 22, 51-65.

Gallwey, T., Drury, C.G., 1986. Task complexity in visual inspection. Human Factors. 28, 595606.

Garrett, S.K., Melloy, B.J., Gramopadhye, A.K., 2001. The effects of per-lot and per-item pacing on inspection performance. International Journal of Industrial Ergonomics. 27, 291302.

Golnabi, H., Asadpour, A., 2007. Design and application of industrial machine vision systems. Robotics and Computer-Integrated Manufacturing. 23 (6), 630-637.

Gramopadhye, A.K., Drury, C.G., Sharit, J., 1997. Feedback strategies for visual search in airframe structural inspection. International Journal of Industrial Ergonomics. 19, 333-344.

Harris, D. H., 1969. The nature of industrial Inspection. Human Factors. 11, 139-148.

Heidl, W., Thumfart, S., Lughofer, E., Eitzinger, C., Klement, E.P., 2013. Machine learning based analysis of gender differences in visual inspection decision making. Information Sciences. 224, 62-76. 
Huang, S.H. Pan, Y.C., 2015. Automated visual inspection in the semiconductor industry: A survey. Computers in Industry. 66, 1-10.

Juran, J., Gryna, F. (Ed.), 1988. Juranís quality control handbook (3rd ed.). McGraw-Hill, New York.

Kirwan, B., Ainsworth, L.K., 1992. A Guide to Task Analysis. Taylor and Francis, Ltd., London.

Kleiner, B.M., Drury, C.G., 1993. Design and evaluation of an inspection training programme. Applied Ergonomics. 24, 75-82.

Kumar, D.P., Kannan, K., 2010. A roadmap for designing an automated visual inspection system. International Journal of Computer Applications. 1 (19), 34-37.

Lam, A., 2000. Tacit knowledge, organizational learning and societal institutions: An integrated framework. Organization studies, 21(3), 487-513.

Laofor, C., Peansupap, V., 2012. Defect detection and quantification system to support subjective visual quality inspection via a digital image processing: A tiling work case study. Automation in Construction. 24, 160-174.

Lin, L., Drury, C. G., Kim, S. W., 2001. Ergonomics and quality in paced assembly lines. Human Factors and Ergonomics in Manufacturing \& Service Industries, 11(4), 377-382.

Lin, H.D., 2007. Computer-aided visual inspection of surface defects in ceramic capacitor chips. Journal of Materials Processing Technology. 189 (1), 19-25.

Lyu, J., Chen, M., 2009. Automated visual inspection expert system for multivariate statistical process control chart. Expert Systems with Applications. 36 (3), 5113-5118.

Mar, N.S.S., Yarlagadda, P.K.D.V., Fookes, C., 2011. Design and development of automatic visual inspection system for PCB manufacturing. Robotics and Computer-Integrated Manufacturing. 27 (5), 949-962.

Mumtaz, R., Mumtaz, M., Mansoor, A.B., Masood, H., 2012. Computer aided visual inspection of aircraft surfaces. International Journal of Image Process, 6 (1), 38-53.

Phipps, D.L., Meakin, G.H., Beatty, P.C.W., 2011. Extending hierarchical task analysis to identify cognitive demands and information design requirements. Applied Ergonomics. 42, 741-748.

Piso, E., 1981. Task analysis for process-control tasks: The method of Annett et al. applied. Journal of Occupational Psychology. 54, 247-254.

Pitts, D. W., Recascino Wise, L., 2010. Workforce diversity in the new millennium: Prospects for research. Review of Public Personnel Administration, 30(1), 44-69.

Rao, P., Bowling, S.R., Khasawneh, M.T., Gramopadhye, A.K., Melloy, B.J., 2006. Impact of Training Standard Complexity on Inspection Performance. Human Factors and Ergonomics in Manufacturing. 16, 109-132. 
Rasmussen, J., 1983. Skills, rules, and knowledge; signals, signs, and symbols, and other distinctions in human performance models. IEEE Transactions on Systems, Man and Cybernetics. 3, 257-266.

Rasmussen, J., 1986. Information Processing and Human-Machine Interaction: An Approach to Cognitive Engineering. North Holland, New York.

Ravikumar, S., Ramachandran, K.I., Sugumaran, V., 2011. Machine learning approach for automated visual inspection of machine components. Expert Systems with Applications. 38 (4), 3260-3266.

Reber, A. S., 1989. Implicit learning and tacit knowledge. Journal of Experimental Psychology: General, 118(3), 219.

See, J.E., 2012. Visual Inspection: A Review of the Literature. Sandia National Laboratories.

See, J. E., Drury, C. G., Speed, A., Williams, A., Khalandi, N., 2017, September. The Role of Visual Inspection in the 21st Century. In Proceedings of the Human Factors and Ergonomics Society Annual Meeting (Vol. 61, No. 1, pp. 262-266). Sage CA: Los Angeles, CA: SAGE Publications.

Smith, E.A., 2001. The role of tacit and explicit knowledge in the workplace. Journal of Knowledge Management. 5 (4), 311-321.

Spitz, G., Drury, C.G., 1978. Inspection of sheet materials - test of model predictions. Human Factors. 20 (5), 521-528.

Sun, J., Sun, Q., Surgenor, B.W., 2012. An adaptable automated visual inspection scheme through online learning. The International Journal of Advanced Manufacturing Technology. 59 (5), 655-667.

Sun, T.H., Tseng, C.C., Chen, M.S., 2010. Electric contacts inspection using machine vision. Image and Vision Computing. 28 (6), 890-901.

Tetteh, E.G., Jiang, S., 2006. The effects of search strategy, task complexity and pacing on visual inspection performance. In The proceedings of the 9th Annual Applied Ergonomics Conference. Orlando, Florida.

Thapa, V.B., Gramopadhye, A.K., Melloy, B., Grimes, L., 1996. Evaluation of different training strategies to improve decision-making performance in inspection. International Journal of Human Factors in Manufacturing. 6, 243-261.

Watts, K.P., 2011. The effect of visual search strategy and overlays on visual inspection of castings. (Master's thesis, Digital Repository@ lowa State University).

Wickens, C., Carswell, C., 2012. Information processing, in G. Salvendy (Eds.), Handbook of Human Factors and Ergonomics (3rd ed.). John Wiley \& Sons, Inc, New Jersey, pp. 117-161. 
2018-08-03

\section{How and why we need to capture tacit} knowledge in manufacturing: Case studies of visual inspection

Johnson, Teegan L.

Elsevier

Johnson T, Fletcher S, and Baker W, Charles R, How and why we need to capture tacit knowledge in manufacturing: Case studies of visual inspection. Applied Ergonomics, Volume 74, Issue January, 2019, pp. 1-9

http://dx.doi.org/10.1016/j.apergo.2018.07.016

Downloaded from Cranfield Library Services E-Repository 